\title{
VIAJANDO ENTRE IDEAS, PERTENENCIAS Y PÚBLICOS
}

\section{TrAVELING BETWEEN IDEAS, PLACES OF BelONGING, AND AUDIENCES}

Bien avanzado este último año de mi programa doctoral, es posible ver los contornos de mi trabajo y reflexionar sobre sus alcances. Así, la invitación a describir el proceso es muy oportuna, pero más allá de las especificidades del programa doctoral y su espacio institucional, quisiera poner el énfasis en los diferentes escenarios, grupos y encuentros que le han dado forma y perspectiva a mi proyecto de investigación y que, a la vez, han sido el "público" al que he dirigido mi trabajo.

La secuencia comienza el Health, Community and Development Research Group ${ }^{1}$, iniciativa de reflexión e intercambio desarrollada por Catherine Campbell $^{2}$ y Flora Cornish ${ }^{3}$, que da cabida a investigaciones cualitativas orientadas a explorar la interacción entre problemas de salud, dinámicas institucionales y respuestas comunitarias en lugares como Tanzanía, India, Nepal, Sudán del Sur y Sudáfrica. En este espacio he comprobado hasta qué punto la "comunidad" juega un papel clave, en términos empíricos y analíticos, en muchos proyectos de salud pública alrededor del mundo, pero como cada caso requiere un conocimiento sobre contextos institucionales e históricos.

Un segundo orden de intercambio es el mundo del activismo en el campo de la salud mental. Tanto en Chile como en Inglaterra he tenido la posibilidad de conocer y compartir experiencias con usuarios, ex-usuarios y sobrevivientes $(\mathrm{u} / \mathrm{e} / \mathrm{s})$ de la psiquiatría, personas que usan su experiencia como fundamento de una crítica radical a la forma en que sistemas de salud en occidente entienden y responden al sufrimiento humano. En Inglaterra este movimiento tiene una larga trayectoria y expresiones muy diversas, una de las cuales es el desarrollo de los "Mad Studies" ${ }^{4}$, una comunidad usuarios y colaboradores que usan la investigación social y el trabajo académico para desarrollar "un proyecto de investigación, producción de conocimiento y acción política dedicada a la crítica y a la superación de formas psicocéntricas de pensamiento, comportamiento, relacionamiento y ' $\operatorname{ser}^{\prime \prime \prime}{ }^{5}$. Sin ser parte de esta red, presentar y debatir mi proyecto con ellas/os ha enriquecido enormemente mi trabajo y ha extendido los alcances de mi investigación.

CRISTIAN R. MONTENEGRO PhD Candidate, Department of Methodology, London School of Economics and Political Sciences https://lse.academia.edu/ CristianRMontenegro
$1 \mathrm{http}: / /$ psych1.Ise.ac.uk/hcd/

2 http://www.Ise.ac.uk/PBS/People/Professor-Catherine-Campbell

3 http://www.Ise.ac.uk/Methodology/People/Academic-Staff/Flora-Cornish/Flora-Cornish

4 https://www.academia.edu/3819953/Introducing_Mad_Studies

5 Menzies, R., LeFrançois, B. A., \& Reaume, G. (2013). Introducing mad studies. In Mad matters: A critical reader in Canadian Mad Studies. Toronto: CSPI. 
A medio camino de mi doctorado tuve un encuentro directo con un tercer público, los "hacedores de políticas", ocasión en la que fui invitado a participar como "fellow" en el Salzburg Global Seminar Session $553^{6}$ Ilamado "Toward a Shared Culture of Health: Enriching and Charting the Patient-Clinician Relationship", en donde también se convocó a médicos, informáticos, expertos en comunicaciones, cientistas sociales y principalmente a pacientes. El encuentro implicó una semana de presentaciones, debates y propuestas y la creación de una comunidad internacional que ha dado seguimiento al desarrollo de esas iniciativas de manera virtual. Actualmente la implementación de una de las propuestas generadas en el encuentro -la simple y transformadora práctica de entregar la ficha clínica a sus "dueños", los pacientes, iniciada en EEUU con el nombre de "Open Notes"7- está en etapa de evaluación en el Complejo Hospitalario Sótero del Río. Es así como esta apasionada colección de perfiles provenientes de diferentes rincones del mundo se ha constituido en un público relevante en mi trabajo. He desarrollado algunas ideas en un texto publicado por el British Medical Journal Opinion ${ }^{8}$.

Finalmente un grupo muy importante han sido los "pares", estudiantes doctorales y/o investigadores jóvenes trabajando en Europa. A principios del año 2016, y con representantes de Brasil, Perú, Colombia y Chile, basados en universidades de Lisboa, París y Londres, nace la idea de la Platform for Social Research on Mental Health in Latin America ${ }^{9}$ o PLASMA, una plataforma de conocimiento mutuo e intercambio para estudiantes doctorales e investigadores jóvenes interesados en utilizar las Ciencias Sociales para entender dinámicas de salud mental en América Latina. Luego del workshop que organizamos en París en junio de $2017^{10}$ estamos organizando una conferencia internacional en Londres, a realizarse en octubre de 2018.

Mi experiencia de estudiar y hacer investigación "afuera" ha estado marcada por una compleja secuencia de espacios de pertenencia e intercambio. Al presentar mis ideas o contrastar otras ideas con la situación chilena he aprendido a reconocer lo distintivo del país y las continuidades y paralelos con lugares inesperados. Lo artículos ${ }^{11}$ que conformarán mi tesis doctoral tienen el objetivo de responder a esta conversación y de comunicar estas especificidades y continuidades a una audiencia amplia, desde cientistas sociales interesados en temas de salud, iniciativas y grupos autónomos de usuarios y aliados, y hacedores de política pública.

\section{Nota de los Editores}

Es importante señalar que esta presentación fue escrita en noviembre de 2017. Cristian obtuvo el grado de Doctor en Métodos de Investigación Social en London School of Economics en octubre de 2018. Su tesis doctoral, titulada "Service-user organisations and the Chilean mental health system: tracing policy expectations and political contestations", puede ser descargada en el siguiente link: http:// etheses.Ise.ac.uk/3806/. En la actualidad, Cristian es Profesor Asistente en la Escuela de Enfermería de la Pontificia Universidad Católica de Chile y asesor en el Departamento de Salud Mental, División de Prevención y Control de Enfermedades, Ministerio de Salud.

\footnotetext{
6 http://www.salzburgglobal.org/calendar/2010-2019/2017/session-553.html

7 https://www.opennotes.org

8 http://blogs.bmj.com/bmj/2017/04/25/cristian-r-montenegro-could-knowing-each-other-in-advance-improve-the-interaction-betweenclinicians-and-patients/

9 https://blogplasma.wordpress.com

10 https://plasmaworkshop2017.wixsite.com/paris

11 El primero de los cuales ya ha sido publicado: Montenegro, C. R., \& Cornish, F. (2017). Historicising involvement: the visibility of user groups in the modernisation of the Chilean Mental Health System. Critical Public Health. http://www.tandfonline.com/doi/abs/10.1080/0 9581596.2017.1400659
} 